\title{
Solving coupled Lane-Emden equations by Green's function and decomposition technique
}

\author{
Randhir Singh* \\ Department of Mathematics, Birla Institute of Technology Mesra, Ranchi-835215, India.
}

\begin{abstract}
In this paper, the Green's function and decomposition technique is proposed for solving the coupled Lane-Emden equations. This approach depends on constructing Green's function before establishing the recursive scheme for the series solution. Unlike, standard Adomian decomposition method, the present method avoids solving a sequence of transcendental equations for the undetermined coefficients. Convergence and error estimation is provided. Three examples of coupled Lane-Emden equations are considered to demonstrate the accuracy of the current algorithm.
\end{abstract}

Keyword: Coupled Lane-Emden equations; Green's function; Adomian decomposition method; Convergence analysis.

\section{Introduction}

This paper aims to extend the application of the Adomian decomposition method with Green's function [1, 2] for solving the following coupled Lane-Emden boundary value problems

$$
\left\{\begin{array}{l}
y_{1}^{\prime \prime}(x)+\frac{\alpha_{1}}{x} y_{1}^{\prime}(x)=f_{1}\left(x, y_{1}(x), y_{2}(x)\right), \quad x \in(0,1) \\
y_{2}^{\prime \prime}(x)+\frac{\alpha_{2}}{x} y_{2}^{\prime}(x)=f_{2}\left(x, y_{1}(x), y_{2}(x)\right), \\
y_{1}^{\prime}(0)=0, \quad y_{2}^{\prime}(0)=0 \\
a_{1} y_{1}(1)+b_{1} y_{1}^{\prime}(1)=c_{1}, \quad a_{2} y_{2}(1)+b_{2} y_{2}^{\prime}(1)=c_{2},
\end{array}\right.
$$

where $a_{1}, a_{2}, b_{1}, b_{2}, c_{1}, c_{2}$ are real constants. In recent years, singular boundary value problems for ordinary differential equations have been studied extensively [1 21] and references therein. However, we find only the following results on coupled Lane-Emden equations.

\footnotetext{
*Corresponding author. E-mail: randhir.math@gmail.com
} 
Recently, in [22 24] authors studied (1.1) with boundary conditions $y_{1}^{\prime}(0)=y_{2}^{\prime}(0)=0$, $y_{1}(1)=y_{2}(1)=1$ and $\alpha_{1}=\alpha_{2}=2$ that relates the concentration of the carbon substrate and the concentration of oxygen. In [25, 26], authors considered the coupled LaneEmden equations (1.1) with boundary conditions $y_{1}^{\prime}(0)=y_{2}^{\prime}(0)=0, y_{1}(1)=1, y_{2}(1)=2$ and $\alpha_{1}=\alpha_{2}=2$ occurs in catalytic diffusion reactions. In [24, 26], the Adomian decomposition method was applied to obtain a convergent analytic approximate solution of (1.1) with $\alpha_{1}=\alpha_{2}=2$. Later, in [27], the variational iteration method was applied to obtain approximations to solutions of (1.1) for shape factors $\alpha_{1}, \alpha_{2}=1,2,3$. In [28], the Sinc-collocation method was used to obtain the solution of (1.1). In [29] authors used the reproducing kernel Hilbert space method for solving to obtain the solution of (1.1).

\section{Adomian decomposition method}

Recently, many researchers 30 36] have applied the Adomian decomposition method to deal with many different scientific models. According to the Adomian decomposition method we rewrite (1.1) in a operator form as

$$
\left\{\begin{array}{l}
L_{1} y_{1}(x)=f_{1}\left(x, y_{1}(x), y_{2}(x)\right), \quad x \in(0,1) \\
L_{2} y_{2}(x)=f_{2}\left(x, y_{1}(x), y_{2}(x)\right)
\end{array}\right.
$$

where $L_{1}=x^{-\alpha_{1}} \frac{d}{d x}\left[x^{\alpha_{1}} \frac{d}{d x}\right]$ and $L_{2}=x^{-\alpha_{2}} \frac{d}{d x}\left[x^{\alpha_{2}} \frac{d}{d x}\right]$ are differential operators and their inverse integral operators are defined as

$$
\left\{\begin{aligned}
L_{1}^{-1}[\cdot] & =\int_{0}^{x} x^{-\alpha_{1}} \int_{0}^{x} x^{\alpha_{1}}[\cdot] d x d x \\
L_{2}^{-1}[\cdot] & =\int_{0}^{x} x^{-\alpha_{2}} \int_{0}^{x} x^{\alpha_{2}}[\cdot] d x d x
\end{aligned}\right.
$$

Operating $L_{1}^{-1}[\cdot], L_{2}^{-1}[\cdot]$ on (2.1) and using $y_{1}^{\prime}(0)=y_{2}^{\prime}(0)=0$, we get

$$
\left\{\begin{array}{l}
y_{1}(x)=y_{1}(0)+L_{1}^{-1}\left[f_{1}\left(x, y_{1}(x), y_{2}(x)\right)\right] \\
y_{2}(x)=y_{2}(0)+L_{2}^{-1}\left[f_{2}\left(x, y_{1}(x), y_{2}(x)\right)\right] .
\end{array}\right.
$$

According to the ADM, we decompose $y_{i}(x)$ and $f_{i}\left(x, y_{1}(x), y_{2}(x)\right)$ as

$$
\begin{cases}y_{1}(x)=\sum_{j=0}^{\infty} y_{1 j}(x), & f_{1}\left(x, y_{1}(x), y_{2}(x)\right)=\sum_{j=0}^{\infty} A_{1 j}, \\ y_{2}(x)=\sum_{j=0}^{\infty} y_{2 j}(x), & f_{2}\left(x, y_{1}(x), y_{2}(x)\right)=\sum_{j=0}^{\infty} A_{2 j},\end{cases}
$$

where $A_{i j}$ are Adomian's polynomials [30] are given

$$
A_{i n}=\frac{1}{n !} \frac{d^{n}}{d \lambda^{n}}\left[f_{i}\left(x, \sum_{j=0}^{\infty} y_{1 j} \lambda^{j}, \sum_{j=0}^{\infty} y_{2 j} \lambda^{j}\right)\right]_{\lambda=0}, \quad i=1,2 .
$$


Substituting (2.4) into (2.3), we get

$$
\left\{\begin{array}{l}
\sum_{j=0}^{\infty} y_{1 j}(x)=y_{1}(0)+L_{1}^{-1}\left[\sum_{j=0}^{\infty} A_{1 j}\right], \\
\sum_{j=0}^{\infty} y_{2 j}(x)=y_{2}(0)+L_{2}^{-1}\left[\sum_{j=0}^{\infty} A_{2 j}\right] .
\end{array}\right.
$$

Upon comparing both sides of (2.6), we have

$$
\left\{\begin{array}{l}
y_{10}(x)=\delta_{1}, \quad y_{20}(x)=\delta_{2}, \\
y_{1 j}\left(x, \delta_{1}, \delta_{2}\right)=L_{1}^{-1}\left[A_{1, j-1}\right], \\
y_{2 j}\left(x, \delta_{1}, \delta_{2}\right)=L_{2}^{-1}\left[A_{2, j-1}\right], \quad j=1,2,3 \ldots
\end{array}\right.
$$

where $y_{1}(0)=\delta_{1}, y_{2}(0)=\delta_{2}$ are unknown constants to be determined. The $n$-term series solutions are given as

$$
\left\{\begin{aligned}
\phi_{1 n}\left(x, \delta_{1}, \delta_{2}\right) & =\sum_{j=0}^{n} y_{1 j}\left(x, \delta_{1}, \delta_{2}\right), \\
\phi_{2 n}\left(x, \delta_{1}, \delta_{2}\right) & =\sum_{j=0}^{n} y_{2 j}\left(x, \delta_{1}, \delta_{2}\right) .
\end{aligned}\right.
$$

The unknown constants may be obtained by imposing boundary condition at $x=1$ on $\phi_{\text {in }}\left(x, \delta_{1}, \delta_{2}\right)$, that leads to

$$
\left\{\begin{array}{c}
a_{1} \phi_{1 n}\left(1, \delta_{1}, \delta_{2}\right)+b_{1} \phi_{1 n}^{\prime}\left(1, \delta_{1}, \delta_{2}\right)-c_{1}=0, \\
a_{2} \phi_{2 n}\left(1, \delta_{1}, \delta_{2}\right)+b_{2} \phi_{2 n}^{\prime}\left(1, \delta_{1}, \delta_{2}\right)-c_{2}=0 .
\end{array}\right.
$$

Solving above transcendental equations for $\delta_{i}$ require additional computational work, and $\delta_{i}$ may not be uniquely determined.

To avoid solving the above sequence of difficult transcendental equations, the Adomian decomposition method with Green's function was introduced in [1, 2]. This technique relies on constructing Green's function before establishing the recursive scheme for the solution components. Unlike the standard Adomian decomposition method, this avoids solving a sequence of transcendental equations for the undetermined coefficients.

\section{Green's function and decomposition technique}

In this section, we extend the application of the Adomian decomposition method with Green's function [2, 12, 37 39], where we transformed the singular boundary value problem into the integral equation before establishing the recursive scheme for the approximate 
solution. To apply this technique to coupled Lane-Emden boundary value problems (1.1), we first consider the equivalent integral form of coupled Lane-Emden equation (1.1) as

$$
\left\{\begin{array}{l}
y_{1}(x)=\frac{c_{1}}{a_{1}}+\int_{0}^{1} G_{1}(x, s) s^{\alpha_{1}} f_{1}\left(s, y_{1}(s), y_{2}(s)\right) d s \\
y_{2}(x)=\frac{c_{2}}{a_{2}}+\int_{0}^{1} G_{2}(x, s) s^{\alpha_{2}} f_{2}\left(s, y_{1}(s), y_{2}(s)\right) d s
\end{array}\right.
$$

where $G_{i}(x, s)$ are given by

$$
G_{i}(x, s)= \begin{cases}\ln s, & x \leq s, \quad \alpha_{i}=1, \quad i=1,2 \\ \ln x, & s \leq x\end{cases}
$$

and

$$
G_{i}(x, s)= \begin{cases}\frac{s^{1-\alpha_{i}}-1}{1-\alpha_{i}}, & x \leq s, \quad \alpha_{i}>1, \quad i=1,2, \\ \frac{x^{1-\alpha_{i}}-1}{1-\alpha_{i}}, & s \leq x .\end{cases}
$$

Substituting the series (2.4) into (3.1), we obtain

$$
\left\{\begin{array}{l}
\sum_{j=0}^{\infty} y_{1 j}(x)=\frac{c_{1}}{a_{1}}+\int_{0}^{1} G_{1}(x, s) s^{\alpha_{1}} \sum_{j=0}^{\infty} A_{1 j} d s \\
\sum_{j=0}^{\infty} y_{2 j}(x)=\frac{c_{2}}{a_{2}}+\int_{0}^{1} G_{2}(x, s) s^{\alpha_{2}} \sum_{j=0}^{\infty} A_{2 j} d s .
\end{array}\right.
$$

Comparing components from both sides of (3.4) we have the following recursive scheme

$$
\left.\begin{array}{l}
y_{10}(x)=\frac{c_{1}}{a_{1}}, \quad y_{20}(x)=\frac{c_{2}}{a_{2}}, \\
y_{1 j}(x)=\int_{0}^{1} G_{1}(x, s) s^{\alpha_{1}} A_{1, j-1} d s, \\
y_{2 j}(x)=\int_{0}^{1} G_{2}(x, s) s^{\alpha_{2}} A_{2, j-1} d s .
\end{array}\right\}
$$

Then, we obtain the approximate series solutions as

$$
\left.\begin{array}{l}
\psi_{1 n}(x)=\sum_{j=0}^{n} y_{1 j}(x), \\
\psi_{2 n}(x)=\sum_{j=0}^{n} y_{2 j}(x) .
\end{array}\right\}
$$

Unlike ADM or MADM, the proposed recursive schemes (3.6) do not require any computation of unknown constants. 


\section{Convergence and error analysis}

Let $E=(C[0,1],\|y\|)$ be a Banach space with norm

$$
\|y\|=\max \left\{\left\|y_{1}\right\|,\left\|y_{2}\right\|\right\}, \quad y \in E,
$$

where, $\left\|y_{1}\right\|=\max _{x \in I=[0,1]}\left|y_{1}(x)\right|$ and $\left\|y_{2}\right\|=\max _{x \in I}\left|y_{2}(x)\right|$.

From (3.5) and (3.6), we have

$$
\Psi_{n}=\sum_{j=0}^{n} \mathbf{y}_{j}(x)=\frac{\mathbf{c}}{\mathbf{a}}+\sum_{j=1}^{n}\left[\int_{0}^{1} \mathbf{G}(x, s) \mathbf{s}^{\alpha} \mathbf{A}_{j-1} d s\right]=\frac{\mathbf{c}}{\mathbf{a}}+\int_{0}^{1} \mathbf{G}(x, s) \mathbf{s}^{\alpha} \sum_{j=1}^{n} A_{j-1} d s,
$$

where

$$
\begin{aligned}
& \mathbf{y}=\left(\begin{array}{l}
y_{1} \\
y_{2}
\end{array}\right), \quad \mathbf{y}_{j}=\left(\begin{array}{c}
y_{1 j} \\
y_{2 j}
\end{array}\right), \mathbf{G}(x, s)=\left(\begin{array}{c}
G_{1}(x, s) \\
G_{2}(x, s)
\end{array}\right), \quad \mathbf{A}_{j}=\left(\begin{array}{c}
A_{1 j} \\
A_{2 j}
\end{array}\right) \\
& \mathbf{f}=\left(\begin{array}{c}
f_{1} \\
f_{2}
\end{array}\right), \quad \mathbf{s}^{\alpha}=\left(\begin{array}{c}
s^{\alpha_{1}} \\
s^{\alpha_{2}}
\end{array}\right), \quad \frac{\mathbf{c}}{\mathbf{a}}=\left(\begin{array}{c}
\frac{c_{1}}{a_{1}} \\
\frac{c 2}{a_{2}}
\end{array}\right), \quad \Psi_{n}=\left(\begin{array}{c}
\psi_{1 n} \\
\psi_{2 n}
\end{array}\right) .
\end{aligned}
$$

Definition 1 . The function $f\left(x, y_{1}, y_{2}\right)$ satisfy Lipschitz condition as

$$
\left|f\left(x, y_{1}, y_{2}\right)-f\left(x, y_{1}^{*}, y_{2}^{*}\right)\right| \leq \sum_{j=1}^{2} l_{j}\left|y_{j}-y_{j}^{*}\right|, \quad \forall\left(x, y_{1}, y_{2}\right), \quad\left(x, y_{1}^{*}, y_{2}^{*}\right) \in D
$$

where $D=\{[0,1] \times R \times R\}$, and $l_{1}, l_{2}$ are Lipschitz constants.

Theorem 4.1. Suppose that the nonlinear function $\mathbf{f}\left(x, y_{1}, y_{2}\right)$ satisfy Lipschitz condition (4.3), then the series solution $\sum_{j=0}^{\infty} \mathbf{y}_{j}$ defined by (3.6) is convergent whenever $\gamma<1$.

Proof. Define

$$
\Psi_{0}=\mathbf{x}_{0}, \Psi_{1}=\mathbf{x}_{0}+\mathbf{x}_{1}, \ldots, \Psi_{n}=\sum_{k=0}^{n} \mathbf{y}_{k}
$$

For $n>m$ and using (4.2), we have

$$
\left\|\Psi_{n}-\Psi_{m}\right\|=\max _{x \in I}\left|\int_{0}^{1} \mathbf{G}(x, s) \mathbf{s}^{\alpha}\left(\sum_{j=1}^{n} \mathbf{A}_{j-1}-\sum_{j=1}^{m} \mathbf{A}_{j-1}\right) d s\right| .
$$

Using $\sum_{j=0}^{n} \mathbf{A}_{j} \leq \mathbf{f}\left(s, \psi_{1 n}, \psi_{2 n}\right)$ from $([40])$, we have

$$
\left\|\Psi_{n}-\Psi_{m}\right\| \leq \max _{x \in I}\left|\int_{0}^{1} \mathbf{G}(x, s) \mathbf{s}^{\alpha}\left[\mathbf{f}\left(s, \psi_{1, n-1}, \psi_{2, n-1}\right)-\mathbf{f}\left(s, \psi_{1, m-1}, \psi_{2, m-1}\right)\right] d s\right| .
$$


Applying the Lipschitz condition, we get

$$
\begin{aligned}
\left\|\Psi_{n}-\Psi_{m}\right\| & \leq \max _{x \in I}\left|\int_{0}^{1} \mathbf{G}(x, s) \mathbf{s}^{\alpha} d s\right| \sum_{i=1}^{2} l_{i} \max _{x \in I}\left|\psi_{i, n-1}-\psi_{i, m-1}\right| \\
& \leq \max _{x \in I}\left|\int_{0}^{1} \mathbf{G}(x, s) \mathbf{s}^{\alpha} d s\right| 2 l \max \left\{\left\|\psi_{1, n-1}-\psi_{1, m-1}\right\|,\left\|\psi_{2, n-1}-\phi_{2, m-1}\right\|\right\} \\
& \leq 2 \mathbf{m} l\left\|\Psi_{n-1}-\Psi_{m-1}\right\|=\delta\left\|\Psi_{n-1}-\Phi_{m-1}\right\|,
\end{aligned}
$$

where

$$
\begin{aligned}
& \mathbf{m}=\max \left\{\max \left|\int_{0}^{1} G_{1}(x, s) s^{\alpha_{1}} d s\right|, \max \left|\int_{0}^{1} G_{2}(x, s) s^{\alpha_{2}} d s\right|\right\}, \\
& l=\max \left\{l_{1}, l_{2}\right\}, \quad \gamma=2 \mathbf{m} l .
\end{aligned}
$$

Thus, we have

$$
\left\|\Psi_{n}-\Psi_{m}\right\| \leq \gamma\left\|\Psi_{n-1}-\Psi_{m-1}\right\| .
$$

By taking $n=m+1$ in (4.5), we see that

$$
\left\|\Psi_{m+1}-\Psi_{m}\right\| \leq \gamma\left\|\Psi_{m}-\Psi_{m-1}\right\| \leq \gamma^{2}\left\|\Psi_{m-1}-\Psi_{m-2}\right\| \leq \ldots \leq \gamma^{m}\left\|\Psi_{1}-\Psi_{0}\right\| .
$$

For all $n, m \in \mathbb{N}$, with $n>m$, consider

$$
\begin{aligned}
& \left\|\Psi_{n}-\Psi_{m}\right\|=\left\|\left(\Psi_{n}-\Psi_{n-1}\right)+\left(\Psi_{n-1}-\Psi_{n-1}\right)+\cdots+\left(\Psi_{m+1}-\Psi_{m}\right)\right\| \\
& \leq\left\|\Psi_{n}-\Psi_{n-1}\right\|+\left\|\Psi_{n-1}-\Psi_{n-2}\right\|+\cdots+\left\|\Psi_{m+1}-\Psi_{m}\right\| \\
& \leq\left[\gamma^{n-1}+\gamma^{n-2}+\cdots+\gamma^{m}\right]\left\|\Psi_{1}-\Psi_{0}\right\| \\
& =\gamma^{m}\left[1+\gamma+\gamma^{2}+\cdots+\gamma^{n-m-1}\right]\left\|\mathbf{y}_{1}\right\| \\
& =\gamma^{m}\left(\frac{1-\gamma^{n-m}}{1-\gamma}\right)\left\|\mathbf{y}_{1}\right\| .
\end{aligned}
$$

It follows that as $\gamma<1$,

$$
\left\|\Psi_{n}-\Psi_{m}\right\| \leq \frac{\gamma^{m}}{1-\gamma}\left\|\mathbf{y}_{1}\right\|
$$

Letting $n, m \rightarrow \infty$, we obtain $\lim _{n, m \rightarrow \infty}\left\|\Psi_{n}-\Psi_{m}\right\|=0$. Hence, $\left\{\Psi_{n}\right\}$ is a Cauchy sequences in the Banach space $E$.

Theorem 4.2. If the approximate solution $\Psi_{n}$ converges to $\mathbf{y}(x)$, then the maximum absolute truncated error is estimated

$$
\left\|\mathbf{y}(x)-\Psi_{m}\right\| \leq \frac{\gamma^{m} \mathbf{m}}{1-\gamma} \max _{x \in I}\left|\mathbf{f}\left(x, y_{10}, y_{20}\right)\right| .
$$


Proof. From (4.6), we have

$$
\left\|\Psi_{n}-\Psi_{m}\right\| \leq \frac{\gamma^{m}}{1-\gamma}\left\|\mathbf{y}_{1}\right\|
$$

Since $\Psi_{n} \rightarrow \mathbf{x}(t)$ as $n \rightarrow \infty$, and the above inequality reduces to

$$
\left\|\mathbf{y}(x)-\Psi_{m}\right\| \leq \frac{\gamma^{m}}{1-\gamma}\left\|\mathbf{y}_{1}\right\|
$$

From (3.5), we have $\mathbf{y}_{1}=\int_{0}^{1} \mathbf{G}(x, s) \mathbf{s}^{\alpha} \mathbf{A}_{0} d s$, and we find

$$
\left\|\mathbf{y}_{1}\right\|=\max _{x \in I}\left|\int_{0}^{1} \mathbf{G}(x, s) \mathbf{s}^{\alpha} \mathbf{A}_{0} d s\right| \leq\left|\mathbf{m} \max _{x \in I}\right| \mathbf{f}\left(x, y_{10}, y_{20}\right) \mid .
$$

Combining (4.8) and (4.9), we obtain error estimate as

$$
\left\|\mathbf{y}(t)-\Psi_{m}\right\| \leq \frac{\gamma^{m} \mathbf{m}}{1-\gamma} \max _{x \in I}\left|\mathbf{f}\left(x, y_{10}, y_{20}\right)\right| .
$$

which completes the proof.

\section{$5 \quad$ Numerical Results}

In this section, we consider three coupled Lane-Emden type boundary value problems to examine the accuracy of the present method. Since the exact solution of the problems is not known, we examine the accuracy and applicability of the present method by the absolute residual error

$$
\left\{\begin{array}{l}
r_{1 n}(x):=\left|\psi_{1 n}^{\prime \prime}(x)+\frac{\alpha_{1}}{x} \psi_{1 n}^{\prime}(x)+f_{1}\left(x, \psi_{1 n}(x), \psi_{2 n}(x)\right)\right|, \quad x \in(0,1), \\
r_{2 n}(x):=\left|\psi_{2 n}^{\prime \prime}(x)+\frac{\alpha_{2}}{x} \psi_{2 n}^{\prime}(x)+f_{2}\left(x, \psi_{1 n}(x), \psi_{2 n}(x)\right)\right|
\end{array}\right.
$$

where $\left[r_{1 n}, r_{2 n}\right]^{T}$ is the absolute residual error and $\left[\psi_{1 n}, \psi_{2 n}\right]^{T}$ is the present approximate solution. The maximum residual errors are defined as

$$
\left\{\begin{aligned}
\operatorname{maxr}_{1 n} & :=\max _{x \in[0,1]} r_{1 n}(x) \\
\operatorname{maxr}_{2 n} & :=\max _{x \in[0,1]} r_{2 n}(x)
\end{aligned}\right.
$$




\subsection{The catalytic diffusion reactions problem [25, 26]}

Example 5.1. Consider the coupled Lane-Emden equation occurs in catalytic diffusion reactions [25, 26] as

$$
\left\{\begin{array}{l}
y_{1}^{\prime \prime}(x)+\frac{2}{x} y_{1}^{\prime}(x)=-k_{1} y_{1}^{2}(x)-k_{2} y_{1}(x) y_{2}(x), \quad x \in(0,1), \\
y_{2}^{\prime \prime}(x)+\frac{2}{x} y_{2}^{\prime}(x)=-k_{3} y_{1}^{2}(x)-k_{4} y_{1}(x) y_{2}(x) \\
y_{1}^{\prime}(0)=0, \quad y_{2}^{\prime}(0)=0, \quad y_{1}(1)=1, \quad y_{2}(1)=2
\end{array}\right.
$$

where the parameters $k_{1}, k_{2}, k_{3}$ and $k_{4}$ are the actual chemical reactions. Here $a_{1}=a_{2}=1$, $b_{1}=b_{2}=0, c_{1}=1$ and $c_{2}=2$.

In Tables 1 and 3, we list the numerical results of the approximate solution and the absolute error obtained by the proposed method of Example 5.1 for $\left(k_{1}=1, k_{2}=2 / 5, k_{3}=\right.$ $\left.1 / 2, k_{4}=1\right)$ and $\left(k_{1}=k_{2}=k_{3}=k_{4}=1 / 2\right)$, respectively. We also compare the numerical results of the maximum residual error $\left[\operatorname{maxr}_{1 n}, \operatorname{maxr}_{2 n}\right]$ obtained by the present method and the results obtained by the modified ADM [26] in Table 2. In Table 4, we list the numerical results of the maximum residual error.

\subsubsection{When $k_{1}=1, k_{2}=2 / 5, k_{3}=1 / 2, k_{4}=1$}

By applying the proposed scheme (3.5) with the initial guesses $y_{10}(x)=1, y_{20}(x)=2$, we obtain the 5-terms series solutions as

$$
\left\{\begin{array}{l}
\psi_{15}(x)=0.776218+0.199501 x^{2}+0.018823 x^{4}+0.005706 x^{6}-0.0003741 x^{8}+0.000125 x^{10} \\
\psi_{25}(x)=1.68423+0.283069 x^{2}+0.0258518 x^{4}+0.007133 x^{6}-0.0004418 x^{8}+0.000152 x^{10}
\end{array} .\right.
$$

Table 1 Numerical results of the approximate solution $\left[\psi_{1 n}(x), \psi_{2 n}(x)\right]$ and the absolute error $\left[r_{1 n}(x), r_{2 n}(x)\right]$ when $k_{1}=1, k_{2}=2 / 5, k_{3}=1 / 2, k_{4}=1$ of Example [5.1]

\begin{tabular}{l|cc|cc|cc|cc}
\hline$x$ & $\psi_{15}(x)$ & $\psi_{25}(x)$ & $r_{15}(x)$ & $r_{25}(x)$ & $\psi_{1,10}(x)$ & $\psi_{2,10}(x)$ & $r_{1,10}(x)$ & $r_{2,10}(x)$ \\
\hline 0.1 & 0.7782151 & 1.6870682 & $7.00 \mathrm{E}-2$ & $8.79 \mathrm{E}-2$ & 0.7836523 & 1.6938487 & $5.51 \mathrm{E}-3$ & $6.84 \mathrm{E}-3$ \\
0.2 & 0.7842287 & 1.6955995 & $6.55 \mathrm{E}-2$ & $8.23 \mathrm{E}-2$ & 0.7893632 & 1.7020042 & $5.10 \mathrm{E}-3$ & $6.34 \mathrm{E}-3$ \\
0.3 & 0.7943299 & 1.7099257 & $5.85 \mathrm{E}-2$ & $7.36 \mathrm{E}-2$ & 0.7989874 & 1.7157379 & $4.48 \mathrm{E}-3$ & $5.57 \mathrm{E}-3$ \\
0.4 & 0.8086434 & 1.7302167 & $4.97 \mathrm{E}-2$ & $6.26 \mathrm{E}-2$ & 0.8126872 & 1.7352661 & $3.71 \mathrm{E}-3$ & $4.63 \mathrm{E}-3$ \\
0.5 & 0.8273577 & 1.7567278 & $3.98 \mathrm{E}-2$ & $5.02 \mathrm{E}-2$ & 0.8306972 & 1.7609008 & $2.89 \mathrm{E}-3$ & $3.62 \mathrm{E}-3$ \\
0.6 & 0.8507387 & 1.7898165 & $2.97 \mathrm{E}-2$ & $3.76 \mathrm{E}-2$ & 0.8533324 & 1.7930602 & $2.10 \mathrm{E}-3$ & $2.64 \mathrm{E}-3$ \\
0.7 & 0.8791464 & 1.8299638 & $2.02 \mathrm{E}-2$ & $2.56 \mathrm{E}-2$ & 0.8809992 & 1.8322829 & $1.39 \mathrm{E}-3$ & $1.75 \mathrm{E}-3$ \\
0.8 & 0.9130553 & 1.8778003 & $1.18 \mathrm{E}-2$ & $1.51 \mathrm{E}-2$ & 0.9142115 & 1.8792486 & $7.90 \mathrm{E}-4$ & $1.01 \mathrm{E}-3$ \\
0.9 & 0.9530791 & 1.9341363 & $5.09 \mathrm{E}-3$ & $6.53 \mathrm{E}-3$ & 0.9536120 & 1.9348042 & $3.30 \mathrm{E}-4$ & $4.29 \mathrm{E}-4$ \\
\hline
\end{tabular}


Table 2 Comparison of the numerical results of the maximum residual error $\left[\operatorname{maxr}_{1 n}, \operatorname{maxr}_{2 n}\right]$ when $k_{1}=1, k_{2}=2 / 5, k_{3}=1 / 2, k_{4}=1$ of Example 5.1

\begin{tabular}{c|cc|cc}
\hline \multirow{2}{*}{$n$} & \multicolumn{2}{|c|}{ The present method } & \multicolumn{2}{c}{ Modified ADM [26] } \\
\cline { 2 - 5 } $\operatorname{maxr}_{1 n}$ & $\operatorname{maxr}_{2 n}$ & $\operatorname{maxr}_{1 n}$ & $\operatorname{maxr}_{2 n}$ \\
\hline 2 & $8.72 \mathrm{E}-2$ & $1.12 \mathrm{E}-1$ & $4.13 \mathrm{E}-1$ & $5.67 \mathrm{E}-1$ \\
3 & $3.95 \mathrm{E}-2$ & $5.05 \mathrm{E}-2$ & $2.36 \mathrm{E}-1$ & $3.09 \mathrm{E}-1$ \\
4 & $2.00 \mathrm{E}-2$ & $2.53 \mathrm{E}-2$ & $6.43 \mathrm{E}-2$ & $8.52 \mathrm{E}-2$ \\
5 & $1.07 \mathrm{E}-2$ & $1.35 \mathrm{E}-2$ & $4.79 \mathrm{E}-2$ & $6.09 \mathrm{E}-2$ \\
6 & $6.01 \mathrm{E}-3$ & $7.58 \mathrm{E}-3$ & $2.09 \mathrm{E}-2$ & $2.51 \mathrm{E}-2$ \\
7 & $3.47 \mathrm{E}-3$ & $4.37 \mathrm{E}-3$ & $1.09 \mathrm{E}-2$ & $1.36 \mathrm{E}-2$ \\
8 & $2.06 \mathrm{E}-3$ & $2.59 \mathrm{E}-3$ & $6.21 \mathrm{E}-3$ & $7.32 \mathrm{E}-3$ \\
9 & $1.24 \mathrm{E}-3$ & $1.56 \mathrm{E}-4$ & $3.35 \mathrm{E}-3$ & $3.28 \mathrm{E}-3$ \\
10 & $7.60 \mathrm{E}-4$ & $9.53 \mathrm{E}-4$ & $1.79 \mathrm{E}-3$ & $2.09 \mathrm{E}-3$ \\
11 & $5.91 \mathrm{E}-4$ & $5.91 \mathrm{E}-4$ & $9.61 \mathrm{E}-4$ & $1.11 \mathrm{E}-3$ \\
\hline
\end{tabular}

5.1.2 When $k_{1}=k_{2}=k_{3}=k_{4}=1 / 2$

On applying the proposed scheme (3.5) with the initial guesses $y_{10}(x)=1, y_{20}(x)=2$, we obtain the 5-terms series solutions as

$\left\{\begin{array}{l}\psi_{15}(x)=0.80364+0.17704 x^{2}+0.017049 x^{4}+0.00223 x^{6}-0.00001 x^{8}+0.0000316 x^{10} \\ \psi_{25}(x)=1.80365+0.17704 x^{2}+0.017049 x^{4}+0.00223 x^{6}-0.00001 x^{8}+0.0000316 x^{10}\end{array}\right.$

Table 3 Numerical results of the approximate solution $\left[\psi_{1 n}(x), \psi_{2 n}(x)\right]$ and the absolute error $\left[r_{1 n}(x), r_{2 n}(x)\right]$ when $k_{1}=k_{2}=k_{3}=k_{4}=1 / 2$ of Example 5.1

\begin{tabular}{l|cc|cc|cc|cc}
\hline$x$ & $\psi_{15}(x)$ & $\psi_{25}(x)$ & $r_{15}(x)$ & $r_{25}(x)$ & $\psi_{1,10}(x)$ & $\psi_{2,10}(x)$ & $r_{1,10}(x)$ & $r_{2,10}(x)$ \\
\hline 0.1 & 0.8054213 & 1.8054213 & $1.42 \mathrm{E}-2$ & $1.42 \mathrm{E}-2$ & 0.8065106 & 1.8065106 & $2.63 \mathrm{E}-4$ & $2.63 \mathrm{E}-4$ \\
0.2 & 0.8107583 & 1.8107583 & $1.33 \mathrm{E}-2$ & $1.33 \mathrm{E}-2$ & 0.8117871 & 1.8117871 & $2.43 \mathrm{E}-4$ & $2.43 \mathrm{E}-4$ \\
0.3 & 0.8197227 & 1.8197227 & $1.18 \mathrm{E}-2$ & $1.18 \mathrm{E}-2$ & 0.8206563 & 1.8206563 & $2.13 \mathrm{E}-4$ & $2.13 \mathrm{E}-4$ \\
0.4 & 0.8324215 & 1.8324215 & $1.00 \mathrm{E}-2$ & $1.00 \mathrm{E}-2$ & 0.8332325 & 1.8332325 & $1.76 \mathrm{E}-4$ & $1.76 \mathrm{E}-4$ \\
0.5 & 0.8490101 & 1.8490101 & $8.05 \mathrm{E}-3$ & $8.05 \mathrm{E}-3$ & 0.8496803 & 1.8496803 & $1.37 \mathrm{E}-4$ & $1.37 \mathrm{E}-4$ \\
0.6 & 0.8696982 & 1.8696982 & $5.98 \mathrm{E}-3$ & $5.98 \mathrm{E}-3$ & 0.8702190 & 1.8702190 & $9.91 \mathrm{E}-5$ & $9.93 \mathrm{E}-5$ \\
0.7 & 0.8947568 & 1.8947568 & $4.05 \mathrm{E}-3$ & $4.05 \mathrm{E}-3$ & 0.8951290 & 1.8951290 & $6.53 \mathrm{E}-5$ & $6.53 \mathrm{E}-5$ \\
0.8 & 0.9245277 & 1.9245277 & $2.36 \mathrm{E}-3$ & $2.36 \mathrm{E}-3$ & 0.9247601 & 1.9247601 & $3.71 \mathrm{E}-5$ & $3.71 \mathrm{E}-5$ \\
0.9 & 0.9594352 & 1.9594352 & $1.00 \mathrm{E}-3$ & $1.01 \mathrm{E}-3$ & 0.9595423 & 1.9595423 & $1.55 \mathrm{E}-5$ & $1.55 \mathrm{E}-5$ \\
\hline
\end{tabular}


Table 4 The numerical results of the maximum residual error $\left[\operatorname{maxr}_{1 n}, \max _{2 n}\right]$ when $k_{1}=$ $k_{2}=k_{3}=k_{4}=1 / 2$ of Example 5.1

\begin{tabular}{c|cc}
\hline$n$ & $\operatorname{maxr}_{1 n}$ & $\operatorname{maxr}_{2 n}$ \\
\hline 2 & $4.15 \mathrm{E}-2$ & $4.15 \mathrm{E}-2$ \\
3 & $1.41 \mathrm{E}-2$ & $1.41 \mathrm{E}-2$ \\
4 & $5.35 \mathrm{E}-3$ & $5.35 \mathrm{E}-3$ \\
5 & $2.15 \mathrm{E}-3$ & $2.15 \mathrm{E}-3$ \\
6 & $9.05 \mathrm{E}-4$ & $9.05 \mathrm{E}-4$ \\
7 & $3.91 \mathrm{E}-4$ & $3.91 \mathrm{E}-4$ \\
8 & $1.73 \mathrm{E}-4$ & $1.73 \mathrm{E}-4$ \\
9 & $7.83 \mathrm{E}-5$ & $7.83 \mathrm{E}-5$ \\
10 & $3.58 \mathrm{E}-5$ & $3.58 \mathrm{E}-5$ \\
11 & $1.66 \mathrm{E}-5$ & $1.66 \mathrm{E}-5$ \\
\hline
\end{tabular}

\subsection{The concentration of the carbon substrate and the concen- tration of oxygen problem [27]}

Example 5.2. Consider the coupled Lane-Emden equations, which was used to study the concentration of the carbon substrate and the concentration of oxygen, as

$$
\left\{\begin{array}{l}
y_{1}^{\prime \prime}(x)+\frac{\alpha_{1}}{x} y_{1}^{\prime}(x)=-b+\frac{a y_{1}(x) y_{2}(x)}{\left(l_{1}+y_{1}\right)\left(m_{1}+y_{2}\right)}+\frac{c y_{1}(x) y_{2}(x)}{\left(l_{2}+y_{1}(x)\right)\left(m_{2}+y_{2}(x)\right)}, \quad x \in(0,1), \\
y_{2}^{\prime \prime}(x)+\frac{\alpha_{2}}{x} y_{2}^{\prime}(x)=\frac{d y_{1}(x) y_{2}(x)}{\left(l_{1}+y_{1}(x)\right)\left(m_{1}+y_{2}(x)\right)}+\frac{e y_{1}(x) y_{2}(x)}{\left(l_{2}+y_{1}(x)\right)\left(m_{2}+y_{2}(x)\right)}, \\
y_{1}^{\prime}(0)=0, \quad y_{1}(1)=1, \quad y_{2}^{\prime}(0)=0, \quad y_{2}(1)=1,
\end{array}\right.
$$

where the parameters $l_{1}=l_{2}=m_{1}=m_{2}=1 / 10000, a=5, b=1, c=d=1 / 10, e=5 / 100$ are fixed as given in [24, 27]. Here, $a_{1}=a_{2}=1, b_{1}=b_{2}=0, c_{1}=c_{2}=1$.

In Table 5, 6 and 7, we list the numerical results of the approximate solution and the absolute error obtained by the proposed method of Example 5.2 for $\left(\alpha_{1}=\alpha_{2}=1\right)$, $\alpha_{1}=\alpha_{2}=2$ and $\alpha_{1}=\alpha_{2}=3$, respectively. We also compare the numerical results of the maximum residual error $\left[\operatorname{maxr}_{1 n}, \operatorname{maxr}_{2 n}\right]$ obtained by the present method and the results obtained by the modified ADM [24] in Table 8 for $\alpha_{1}=\alpha_{2}=2$.

\subsubsection{When the shape factors $\alpha_{1}=\alpha_{2}=1$}

By applying the proposed scheme (3.5) with the initial guesses $y_{10}(x)=1, y_{20}(x)=1$, we obtain the 4-terms series solutions as

$$
\left\{\begin{array}{l}
\psi_{14}(x)=2.02484-1.02488 x^{2}+0.00006968 x^{4}-0.0000308 x^{6}+8.57 \times 10^{-6} x^{8} \\
\psi_{24}(x)=1.0375-0.0374966 x^{2}+2.049 \times 10^{-6} x^{4}-9.06 \times 10^{-7} x^{6}+2.52 \times 10^{-7} x^{8}
\end{array}\right.
$$


Table 5 Numerical results of the approximate solution $\left[\psi_{1 n}(x), \psi_{2 n}(x)\right]$ and the absolute error $\left[r_{1 n}(x), r_{2 n}(x)\right]$ when $\alpha_{1}=\alpha_{2}=1$ of Example 5.2

\begin{tabular}{l|cc|cc|cc|cc}
\hline$x$ & $\psi_{12}(x)$ & $\psi_{22}(x)$ & $r_{12}(x)$ & $r_{22}(x)$ & $\psi_{14}(x)$ & $\psi_{24}(x)$ & $r_{14}(x)$ & $r_{24}(x)$ \\
\hline 0.1 & 2.0145977 & 1.0371205 & $2.61 \mathrm{E}-4$ & $7.68 \mathrm{E}-6$ & 2.0145872 & 1.0371202 & $2.67 \mathrm{E}-4$ & $7.87 \mathrm{E}-6$ \\
0.2 & 1.9838514 & 1.0359956 & $2.49 \mathrm{E}-4$ & $7.33 \mathrm{E}-6$ & 1.9838408 & 1.0359953 & $2.40 \mathrm{E}-4$ & $7.07 \mathrm{E}-6$ \\
0.3 & 1.9326076 & 1.0341208 & $2.29 \mathrm{E}-4$ & $6.76 \mathrm{E}-6$ & 1.9325971 & 1.0341205 & $1.99 \mathrm{E}-4$ & $5.86 \mathrm{E}-6$ \\
0.4 & 1.8608665 & 1.0314960 & $2.03 \mathrm{E}-4$ & $5.98 \mathrm{E}-6$ & 1.8608563 & 1.0314957 & $1.50 \mathrm{E}-4$ & $4.42 \mathrm{E}-6$ \\
0.5 & 1.7686285 & 1.0281214 & $1.70 \mathrm{E}-4$ & $5.01 \mathrm{E}-6$ & 1.7686191 & 1.0281211 & $1.00 \mathrm{E}-4$ & $2.95 \mathrm{E}-6$ \\
0.6 & 1.6558940 & 1.0239968 & $1.32 \mathrm{E}-4$ & $3.90 \mathrm{E}-6$ & 1.6558857 & 1.0239966 & $5.69 \mathrm{E}-5$ & $1.67 \mathrm{E}-6$ \\
0.7 & 1.5226632 & 1.0191224 & $9.16 \mathrm{E}-5$ & $2.69 \mathrm{E}-6$ & 1.5226567 & 1.0191222 & $2.49 \mathrm{E}-5$ & $7.33 \mathrm{E}-7$ \\
0.8 & 1.3689369 & 1.0134981 & $5.07 \mathrm{E}-5$ & $1.49 \mathrm{E}-6$ & 1.3689325 & 1.0134980 & $6.88 \mathrm{E}-6$ & $2.02 \mathrm{E}-7$ \\
0.9 & 1.1947156 & 1.0071239 & $1.61 \mathrm{E}-5$ & $4.76 \mathrm{E}-7$ & 1.1947134 & 1.0071239 & $6.08 \mathrm{E}-7$ & $1.78 \mathrm{E}-8$ \\
\hline
\end{tabular}

\subsubsection{For shape factors $\alpha_{1}=\alpha_{2}=2$}

Using the proposed scheme (3.5) with the initial guesses $y_{10}(x)=1, y_{20}(x)=1$, we obtain the 4-terms series solutions as

$$
\left\{\begin{array}{l}
\psi_{14}(x)=1.66653-0.666545 x^{2}+0.0000172 x^{4}-5.2743 \times 10^{-6} x^{6}+2.054 \times 10^{-6} x^{8} \\
\psi_{24}(x)=1.025-0.0249964 x^{2}+5.172 \times 10^{-7} x^{4}-1.582 \times 10^{-7} x^{6}+6.164 \times 10^{-8} x^{8}
\end{array} .\right.
$$

Table 6 Numerical results of the approximate solution $\left[\psi_{1 n}(x), \psi_{2 n}(x)\right]$ and the absolute error $\left[r_{1 n}(x), r_{2 n}(x)\right]$ when $\alpha_{1}=\alpha_{2}=2$ of Example 5.2

\begin{tabular}{l|cc|cc|cc|cc}
\hline$x$ & $\psi_{12}(x)$ & $\psi_{22}(x)$ & $r_{12}(x)$ & $r_{22}(x)$ & $\psi_{14}(x)$ & $\psi_{24}(x)$ & $r_{14}(x)$ & $r_{24}(x)$ \\
\hline 0.1 & 1.6598747 & 1.0247462 & $1.31 \mathrm{E}-4$ & $3.94 \mathrm{E}-6$ & 1.6598657 & 1.0247459 & $5.70 \mathrm{E}-5$ & $1.71 \mathrm{E}-6$ \\
0.2 & 1.6398780 & 1.0239963 & $1.25 \mathrm{E}-4$ & $3.75 \mathrm{E}-6$ & 1.6398694 & 1.0239960 & $5.10 \mathrm{E}-5$ & $1.53 \mathrm{E}-6$ \\
0.3 & 1.6065503 & 1.0227465 & $1.14 \mathrm{E}-4$ & $3.44 \mathrm{E}-6$ & 1.6065422 & 1.0227462 & $4.20 \mathrm{E}-5$ & $1.26 \mathrm{E}-6$ \\
0.4 & 1.5598915 & 1.0209967 & $1.00 \mathrm{E}-4$ & $3.01 \mathrm{E}-6$ & 1.5598843 & 1.0209965 & $3.14 \mathrm{E}-5$ & $9.43 \mathrm{E}-7$ \\
0.5 & 1.4999020 & 1.0187470 & $8.34 \mathrm{E}-5$ & $2.50 \mathrm{E}-6$ & 1.4998959 & 1.0187468 & $2.07 \mathrm{E}-5$ & $6.23 \mathrm{E}-7$ \\
0.6 & 1.4265818 & 1.0159974 & $6.38 \mathrm{E}-5$ & $1.91 \mathrm{E}-6$ & 1.4265769 & 1.0159973 & $1.15 \mathrm{E}-5$ & $3.47 \mathrm{E}-7$ \\
0.7 & 1.3399312 & 1.0127479 & $4.31 \mathrm{E}-5$ & $1.29 \mathrm{E}-6$ & 1.3399276 & 1.0127478 & $4.97 \mathrm{E}-6$ & $1.49 \mathrm{E}-7$ \\
0.8 & 1.2399505 & 1.0089985 & $2.32 \mathrm{E}-5$ & $6.97 \mathrm{E}-7$ & 1.2399482 & 1.0089984 & $1.33 \mathrm{E}-6$ & $4.00 \mathrm{E}-8$ \\
0.9 & 1.1266400 & 1.0047492 & $7.12 \mathrm{E}-6$ & $2.13 \mathrm{E}-7$ & 1.1266389 & 1.0047491 & $1.13 \mathrm{E}-7$ & $3.40 \mathrm{E}-9$ \\
\hline
\end{tabular}

\subsubsection{For shape factors $\alpha_{1}=\alpha_{2}=3$}

Making use of the proposed scheme (3.5) with the initial guesses $y_{10}(x)=1, y_{20}(x)=1$, we obtain the 4-terms series solutions as

$\left\{\begin{array}{l}\psi_{14}(x)=1.49989-0.4999 x^{2}+8.1818 \times 10^{-6} x^{4}-1.295 \times 10^{-6} x^{6}+7.802 \times 10^{-7} x^{8}, \\ \psi_{24}(x)=1.01875-0.018747 x^{2}+2.454 \times 10^{-7} x^{4}-3.886 \times 10^{-8} x^{6}+2.340 \times 10^{-8} x^{8} .\end{array}\right.$ 
Table 7 Numerical results of the approximate solution $\left[\psi_{1 n}(x), \psi_{2 n}(x)\right]$ and the absolute error $\left[r_{1 n}(x), r_{2 n}(x)\right]$ when $\alpha_{1}=\alpha_{2}=3$ of Example 5.2

\begin{tabular}{l|cc|cc|cc|cc}
\hline$x$ & $\psi_{12}(x)$ & $\psi_{22}(x)$ & $r_{12}(x)$ & $r_{22}(x)$ & $\psi_{14}(x)$ & $\psi_{24}(x)$ & $r_{14}(x)$ & $r_{24}(x)$ \\
\hline 0.1 & 1.4948975 & 1.0185594 & $8.20 \mathrm{E}-5$ & $2.46 \mathrm{E}-6$ & 1.4948929 & 1.0185592 & $2.00 \mathrm{E}-5$ & $6.01 \mathrm{E}-7$ \\
0.2 & 1.4799003 & 1.0179970 & $7.79 \mathrm{E}-5$ & $2.33 \mathrm{E}-6$ & 1.4798959 & 1.0179968 & $1.79 \mathrm{E}-5$ & $5.37 \mathrm{E}-7$ \\
0.3 & 1.4549050 & 1.0170596 & $7.12 \mathrm{E}-5$ & $2.13 \mathrm{E}-6$ & 1.4549010 & 1.0170595 & $1.47 \mathrm{E}-5$ & $4.41 \mathrm{E}-7$ \\
0.4 & 1.4199117 & 1.0157473 & $6.21 \mathrm{E}-5$ & $1.86 \mathrm{E}-6$ & 1.4199081 & 1.0157472 & $1.09 \mathrm{E}-5$ & $3.28 \mathrm{E}-7$ \\
0.5 & 1.3749204 & 1.0140601 & $5.11 \mathrm{E}-5$ & $1.53 \mathrm{E}-6$ & 1.3749175 & 1.0140600 & $7.17 \mathrm{E}-6$ & $2.15 \mathrm{E}-7$ \\
0.6 & 1.3199313 & 1.0119979 & $3.88 \mathrm{E}-5$ & $1.16 \mathrm{E}-6$ & 1.3199290 & 1.0119978 & $3.96 \mathrm{E}-6$ & $1.18 \mathrm{E}-7$ \\
0.7 & 1.2549445 & 1.0095608 & $2.59 \mathrm{E}-5$ & $7.77 \mathrm{E}-7$ & 1.2549429 & 1.0095607 & $1.68 \mathrm{E}-6$ & $5.04 \mathrm{E}-8$ \\
0.8 & 1.1799602 & 1.0067488 & $1.37 \mathrm{E}-5$ & $4.12 \mathrm{E}-7$ & 1.1799593 & 1.0067487 & $4.43 \mathrm{E}-7$ & $1.33 \mathrm{E}-8$ \\
0.9 & 1.0949786 & 1.0035618 & $4.12 \mathrm{E}-6$ & $1.23 \mathrm{E}-7$ & 1.0949782 & 1.0035618 & $3.69 \mathrm{E}-8$ & $1.10 \mathrm{E}-9$ \\
\hline
\end{tabular}

Table 8 Comparison of the numerical results of the maximum residual error $\left[\operatorname{maxr}_{1 n}, \operatorname{maxr}_{2 n}\right]$ when $\alpha_{1}=\alpha_{2}=2$ of Example 5.2

\begin{tabular}{c|cc|cc}
\hline \multirow{2}{*}{$n$} & \multicolumn{2}{|c|}{ The present method } & \multicolumn{2}{c}{ Modified ADM [24] } \\
\cline { 2 - 5 } & $\operatorname{maxr}_{1 n}$ & maxr $_{2 n}$ & maxr $_{1 n}$ & maxr $_{2 n}$ \\
\hline 2 & $1.33 \mathrm{E}-4$ & $4.01 \mathrm{E}-6$ & $1.18 \mathrm{E}-3$ & $3.48 \mathrm{E}-5$ \\
3 & $8.87 \mathrm{E}-5$ & $2.66 \mathrm{E}-6$ & $8.53 \mathrm{E}-4$ & $2.51 \mathrm{E}-5$ \\
4 & $5.91 \mathrm{E}-5$ & $1.77 \mathrm{E}-6$ & $6.21 \mathrm{E}-4$ & $1.82 \mathrm{E}-5$ \\
5 & $3.94 \mathrm{E}-5$ & $1.18 \mathrm{E}-6$ & $4.52 \mathrm{E}-4$ & $1.33 \mathrm{E}-5$ \\
6 & $2.62 \mathrm{E}-5$ & $7.87 \mathrm{E}-7$ & $3.29 \mathrm{E}-4$ & $9.69 \mathrm{E}-6$ \\
7 & $1.74 \mathrm{E}-5$ & $5.25 \mathrm{E}-7$ & $2.39 \mathrm{E}-4$ & $7.06 \mathrm{E}-6$ \\
\hline
\end{tabular}




\subsection{The steady-state concentrations of $\mathrm{CO}_{2}$ and PGE [41]}

Example 5.3. We consider the system of nonlinear differential equations, which arising in the study of the steady-state concentrations of $\mathrm{CO}_{2}$ and PGE as

$$
\left\{\begin{array}{l}
y_{1}^{\prime \prime}(x)=\frac{\alpha_{1} y_{1}(x) y_{2}(x)}{1+\beta_{1} y_{1}(x)+\beta_{2} y_{2}(x)}, \quad x \in(0,1), \\
y_{2}^{\prime \prime}(x)=\frac{\alpha_{2} y_{1}(x) y_{2}(x)}{1+\beta_{1} y_{1}(x)+\beta_{2} y_{2}(x)}, \\
y_{1}(0)=1, \quad y_{1}(1)=k, \quad y_{2}^{\prime}(0)=0, \quad y_{2}(1)=1,
\end{array}\right.
$$

where the constants $\alpha_{1}, \alpha_{2}, \beta_{1}, \beta_{2}, k$ are normalized parameters, $x$ is the dimensionless distance as measured from the center, and $k$ is the dimensionless concentration of $\mathrm{CO}_{2}$ at the surface of the catalyst [41]. We fix the parameters $\alpha_{1}=1, \alpha_{2}=2, \beta_{1}=1, \beta_{2}=$ $3, k=0.5$ as in [41]. By applying the proposed scheme, we obtain the approximate series solutions as

$$
\left\{\begin{array}{c}
\psi_{14}(x)=1-0.58005 x+0.09292 x^{2}-0.01397 x^{3}+0.00173 x^{4}-0.000703 x^{5}+0.000057 x^{6} \\
+0.00001590 x^{7}+8.1396 \times 10^{-7} x^{8} \\
\psi_{24}(x)=0.83989+0.185843 x^{2}-0.027952 x^{3}+0.003475 x^{4}-0.0014068 x^{5}+0.0001145 x^{6} \\
+0.0000318 x^{7}+1.627 \times 10^{-6} x^{8}
\end{array}\right.
$$

In Table 9, we list the numerical results of the approximate solution and the absolute error obtained by the proposed method of Example 5.3. We also compare the numerical results of the maximum residual error $\left[\operatorname{maxr}_{1 n}, \operatorname{maxr}_{2 n}\right]$ obtained by the present method and the results obtained by the numerical method [42] in Table 10.

Table 9 Numerical results of the approximate solution $\left[\psi_{1 n}(x), \psi_{2 n}(x)\right]$ and the absolute error $\left[r_{1 n}(x), r_{2 n}(x)\right]$ when $\alpha_{1}=1, \alpha_{2}=2, \beta_{1}=1, \beta_{2}=3, k=0.5$ of Example 5.3

\begin{tabular}{l|ll|ll|ll|lc}
\hline$x$ & $\psi_{12}(x)$ & $\psi_{22}(x)$ & $r_{12}(x)$ & $r_{22}(x)$ & $\psi_{14}(x)$ & $\psi_{24}(x)$ & $r_{14}(x)$ & $r_{24}(x)$ \\
\hline 0.1 & 0.9428976 & 0.8415025 & $1.30 \mathrm{E}-4$ & $2.60 \mathrm{E}-4$ & 0.9429113 & 0.8417515 & $8.59 \mathrm{E}-7$ & $1.71 \mathrm{E}-6$ \\
0.2 & 0.8875704 & 0.8468806 & $4.90 \mathrm{E}-5$ & $9.80 \mathrm{E}-5$ & 0.8875994 & 0.8471355 & $3.27 \mathrm{E}-7$ & $6.55 \mathrm{E}-7$ \\
0.3 & 0.8339413 & 0.8556549 & $2.27 \mathrm{E}-4$ & $4.55 \mathrm{E}-4$ & 0.8339853 & 0.8559153 & $1.40 \mathrm{E}-6$ & $2.81 \mathrm{E}-6$ \\
0.4 & 0.7819361 & 0.8676770 & $4.08 \mathrm{E}-4$ & $8.17 \mathrm{E}-4$ & 0.7819931 & 0.8679387 & $2.39 \mathrm{E}-6$ & $4.78 \mathrm{E}-6$ \\
0.5 & 0.7314823 & 0.8828019 & $5.87 \mathrm{E}-4$ & $1.17 \mathrm{E}-3$ & 0.7315485 & 0.8830574 & $3.14 \mathrm{E}-6$ & $6.28 \mathrm{E}-6$ \\
0.6 & 0.6825087 & 0.9008874 & $7.54 \mathrm{E}-4$ & $1.50 \mathrm{E}-3$ & 0.6825785 & 0.9011254 & $3.36 \mathrm{E}-6$ & $6.73 \mathrm{E}-6$ \\
0.7 & 0.6349449 & 0.9217922 & $8.93 \mathrm{E}-4$ & $1.78 \mathrm{E}-3$ & 0.6350110 & 0.9219982 & $2.63 \mathrm{E}-6$ & $5.26 \mathrm{E}-6$ \\
0.8 & 0.5887200 & 0.9453750 & $9.81 \mathrm{E}-4$ & $1.96 \mathrm{E}-3$ & 0.5887737 & 0.9455316 & $4.55 \mathrm{E}-7$ & $9.10 \mathrm{E}-7$ \\
0.9 & 0.5437627 & 0.9714928 & $9.91 \mathrm{E}-4$ & $1.98 \mathrm{E}-3$ & 0.5437943 & 0.9715808 & $3.48 \mathrm{E}-6$ & $6.97 \mathrm{E}-6$ \\
\hline
\end{tabular}


Table 10 Comparison of the numerical results of the maximum residual errors $\left[\operatorname{maxr}_{1 n}, \operatorname{maxr}_{2 n}\right]$ when $\alpha_{1}=1, \alpha_{2}=2, \beta_{1}=1, \beta_{2}=3, k=0.5$ of Example 5.3

\begin{tabular}{l|ll|ll}
\hline \multirow{2}{*}{$n$} & \multicolumn{2}{|c|}{ The present method } & \multicolumn{2}{c}{ Method in [42] } \\
\cline { 2 - 5 } $\operatorname{maxr}_{1 n}$ & $\operatorname{maxr}_{2 n}$ & $\operatorname{maxr}_{1 n}$ & $\operatorname{maxr}_{2 n}$ \\
\hline 2 & $4.36 \mathrm{E}-3$ & $8.71 \mathrm{E}-3$ & $3.86 \mathrm{E}-2$ & $7.73 \mathrm{E}-2$ \\
3 & $9.99 \mathrm{E}-4$ & $2.00 \mathrm{E}-3$ & $1.14 \mathrm{E}-3$ & $2.28 \mathrm{E}-3$ \\
4 & $3.54 \mathrm{E}-5$ & $7.07 \mathrm{E}-5$ & $4.87 \mathrm{E}-4$ & $9.75 \mathrm{E}-4$ \\
5 & $3.38 \mathrm{E}-6$ & $6.77 \mathrm{E}-6$ & $4.07 \mathrm{E}-5$ & $8.14 \mathrm{E}-5$ \\
6 & $5.29 \mathrm{E}-7$ & $1.06 \mathrm{E}-6$ & $1.01 \mathrm{E}-6$ & $2.03 \mathrm{E}-6$ \\
7 & $1.05 \mathrm{E}-7$ & $2.10 \mathrm{E}-7$ & $3.90 \mathrm{E}-7$ & $7.81 \mathrm{E}-7$ \\
8 & $2.76 \mathrm{E}-8$ & $5.52 \mathrm{E}-8$ & $4.88 \mathrm{E}-7$ & $9.76 \mathrm{E}-9$ \\
9 & $2.80 \mathrm{E}-9$ & $5.61 \mathrm{E}-9$ & $3.64 \mathrm{E}-9$ & $7.28 \mathrm{E}-9$ \\
10 & $2.59 \mathrm{E}-10$ & $5.17 \mathrm{E}-10$ & $1.22 \mathrm{E}-10$ & $2.43 \mathrm{E}-10$ \\
\hline
\end{tabular}

\section{Concluding remarks}

The theory of coupled Lane-Emden equation finds its vital presence in many of the natural or physical processes such as occurs in catalytic diffusion reactions [26], and some coupled Lane-Emden equations that relate the concentration of the carbon substrate and the concentration of oxygen [24]. An analytical approach has been presented for the approximate series solution of the coupled Lane-Emden equation. Unlike the standard Adomian decomposition method, the proposed technique does not require the computation of unknown constants. Unlike the numerical methods, our approach does not require any linearization or discretization of variables. Convergence and error estimation of the method is provided.

\section{References}

[1] Singh, R., Kumar, J., Nelakanti, G.: Nelakanti, Numerical solution of singular boundary value problems using Green's function and improved decomposition method, Journal of Applied Mathematics and Computing 43 (1-2) (2013) 409-425.

[2] Singh, R., Kumar, J.: An efficient numerical technique for the solution of nonlinear singular boundary value problems, Computer Physics Communications 185 (4) (2014) 1282-1289.

[3] Inc, M., Evans, D.: The decomposition method for solving of a class of singular two-point boundary value problems, International Journal of Computer Mathematics 80 (7) (2003) 869-882.

[4] Inc, M., Ergut, M., Cherruault, Y.: A different approach for solving singular two-point boundary value problems, Kybernetes: The International Journal of Systems \& Cybernetics 34 (7) (2005) 934-940.

[5] Mittal, R., Nigam, R.: Solution of a class of singular boundary value problems, Numerical Algorithms 47 (2) (2008) 169-179. 
[6] Wazwaz, A.M., Rach, R.: Comparison of the Adomian decomposition method and the variational iteration method for solving the Lane-Emden equations of the first and second kinds, Kybernetes 40 (9/10) (2011) 1305-1318.

[7] Wazwaz, A.M.: The variational iteration method for solving systems of equations of Emden-Fowler type, International Journal of Computer Mathematics 88 (16) (2011) 34063415 .

[8] Khuri, S., Sayfy, A.: A novel approach for the solution of a class of singular boundary value problems arising in physiology, Mathematical and Computer Modelling 52 (3) (2010) 626636.

[9] Ebaid, A.: A new analytical and numerical treatment for singular two-point boundary value problems via the Adomian decomposition method, Journal of Computational and Applied Mathematics 235 (8) (2011) 1914-1924.

[10] Wazwaz, A.M., Rach, R., Duan, J.S.: Adomian decomposition method for solving the volterra integral form of the Lane-Emden equations with initial values and boundary conditions, Applied Mathematics and Computation 219 (10) (2013) 5004-5019.

[11] Kumar, M., Singh, N.: Modified Adomian decomposition method and computer implementation for solving singular boundary value problems arising in various physical problems, Computers \& Chemical Engineering 34 (11) (2010) 1750-1760.

[12] Singh, R., Kumar, J.: The Adomian decomposition method with Green's function for solving nonlinear singular boundary value problems, Journal of Applied Mathematics and Computing 44 (1-2) (2014) 397-416.

[13] Singh, M., Verma, A.K.: An effective computational technique for a class of Lane-Emden equations, Journal of Mathematical Chemistry 54 (1) (2016) 231-251.

[14] Das, N., Singh, R., Wazwaz, A.M., Kumar, J.: An algorithm based on the variational iteration technique for the Bratu-type and the Lane-Emden problems, Journal of Mathematical Chemistry 54 (2) (2016) 527-551.

[15] Singh, R., Das, N., Kumar, J.: The optimal modified variational iteration method for the Lane-Emden equations with Neumann and Robin boundary conditions, The European Physical Journal Plus 132 (6) (2017) 251.

[16] Singh, R.: Optimal homotopy analysis method for the non-isothermal Reaction-Diffusion model equations in a spherical catalyst, Journal of Mathematical Chemistry 56 (2018) $2579-2590$.

[17] Singh, R.: Analytical approach for computation of exact and analytic approximate solutions to the system of Lane-Emden-fowler type equations arising in astrophysics, The European Physical Journal Plus 133 (8) (2018) 320.

[18] Singh, R., Garg, H., Guleria, V.: Haar wavelet collocation method for Lane-Emden equations with Dirichlet, Neumann and Neumann-Robin boundary conditions, Journal of Computational and Applied Mathematics 346 (2019) 150-161. 
[19] Singh, R.: A modified homotopy perturbation method for nonlinear singular Lane-Emden equations arising in various physical models, International Journal of Applied and Computational Mathematics 5 (3) (2019) 64.

[20] Singh, R., Guleria, V., Singh, M.: Haar wavelet quasilinearization method for numerical solution of Emden-Fowler type equations, Mathematics and Computers in Simulation, (2020), doi: https://doi.org/10.1016/j.matcom.2020.02.004.

[21] Singh, R.: Analytic solution of singular Emden-Fowler-type equations by Green's function and homotopy analysis method, The European Physical Journal Plus 134 (11) (2019) 583.

[22] Muthukumar, S., Veeramuni, M., Lakshmanan, R.: Analytical expression of concentration of substrate and oxygen in excess sludge production using adomian decomposition method, Indian J. Appl. Res 4 (2014) 387-391.

[23] Wazwaz, A.M., Rach, R., Duan, J.S.: A study on the systems of the volterra integral forms of the Lane-Emden equations by the Adomian decomposition method, Mathematical Methods in the Applied Sciences 37 (1) (2014) 10-19.

[24] Duan, J.S., Rach, R. , Wazwaz, A.M.: Oxygen and carbon substrate concentrations in microbial floc particles by the Adomian decomposition method, MATCH Commun. Math. Comput. Chem 73 (2015) 785-796.

[25] Flockerzi, D., Sundmacher, K.: On coupled Lane-Emden equations arising in dusty fluid models, in: Journal of Physics: Conference Series, Vol. 268, IOP Publishing, 2011, p. 012006 .

[26] Rach, R., Duan, J.S., Wazwaz, A.M.: Solving coupled Lane-Emden boundary value problems in catalytic diffusion reactions by the Adomian decomposition method, Journal of Mathematical Chemistry 52 (1) (2014) 255-267.

[27] Wazwaz, A.M., Rach, R., Duan, J.S.: Variational iteration method for solving oxygen and carbon substrate concentrations in microbial floc particles, MATCH Commun. Math. Comput. Chem 76 (2016) 511-523.

[28] Saadatmandi, A., Fayyaz, S.: Numerical study of oxygen and carbon substrate concentrations in excess sludge production using sinc-collocation method, MATCH Commun. Math. Comput. Chem 80 (2018) 355-368.

[29] Hao, T., Song, S.: Solving coupled Lane-Emden equations arising in catalytic diffusion reactions by reproducing kernel hilbert space method, in: 2015 International conference on Applied Science and Engineering Innovation, Atlantis Press, 2015.

[30] Adomian, G., Rach, R.: Inversion of nonlinear stochastic operators, Journal of Mathematical Analysis and Applications 91 (1) (1983) 39-46.

[31] Adomian, G., Rach, R.: A new algorithm for matching boundary conditions in decomposition solutions, Applied Mathematics and Computation 57 (1) (1993) 61-68.

[32] Adomian, G., Rach, R.: Modified decomposition solution of linear and nonlinear boundaryvalue problems, Nonlinear Analysis: Theory, Methods \& Applications 23 (5) (1994) 615619. 
[33] Adomian, G.: Solving Frontier Problems of Physics: The Decomposition Method, Kluwer Academic Publishers, 1994.

[34] Wazwaz, A.M.: Approximate solutions to boundary value problems of higher order by the modified decomposition method, Computers \& Mathematics with Applications 40 (6-7) (2000) 679-691.

[35] Wazwaz, A.M.: A reliable algorithm for obtaining positive solutions for nonlinear boundary value problems, Computers \& Mathematics with Applications 41 (10-11) (2001) 1237-1244.

[36] Jang, B.: Two-point boundary value problems by the extended Adomian decomposition method, Journal of Computational and Applied Mathematics 219 (1) (2008) 253-262.

[37] Singh, R., Kumar, J., Nelakanti,G.: Approximate series solution of fourth-order boundary value problems using decomposition method with Green's function, Journal of Mathematical Chemistry 52 (4) (2014) 1099-1118.

[38] Singh, R., Wazwaz, A.M.: Numerical solutions of fourth-order volterra integro-differential equations by the Green,s function and decomposition method, Mathematical Sciences 10 (4) (2016) 159-166.

[39] Singh, R., Wazwaz, A.M., Kumar, J.: An efficient semi-numerical technique for solving nonlinear singular boundary value problems arising in various physical models, International Journal of Computer Mathematics 93 (8) (2016) 1330-1346.

[40] Rach, R.: A new definition of the Adomian polynomials, Kybernetes 37 (7) (2008) 910-955.

[41] Duan, J.S., Rach, R., Wazwaz, A.M.: Steady-state concentrations of carbon dioxide absorbed into phenyl glycidyl ether solutions by the adomian decomposition method, Journal of Mathematical Chemistry 53 (4) (2015) 1054-1067.

[42] Hao, T.C., Cong, F.Z., Shang, Y.F.: An efficient method for solving coupled lane-emden boundary value problems in catalytic diffusion reactions and error estimate, Journal of Mathematical Chemistry 56 (9) (2018) 2691-2706. 